\title{
Quespillo et ses compagnons : la figure du gracioso dans le théâtre quechua colonial
}

Quespillo y sus compañeros: la figura del gracioso en el teatro quechua colonial Quespillo and his companions: the character of the gracioso in the Quechua Colonial Theatre

\section{Rossella Martin}

\section{OpenEdition}

Journals

Édition électronique

URL : http://journals.openedition.org/bifea/5257

DOI : $10.4000 /$ bifea.5257

ISSN : 2076-5827

\section{Éditeur}

Institut Français d'Études Andines

\section{Édition imprimée}

Date de publication : 1 août 2014

Pagination : 311-333

ISSN : 0303-7495

\section{Référence électronique}

Rossella Martin, «Quespillo et ses compagnons : la figure du gracioso dans le théâtre quechua colonial », Bulletin de l'Institut français d'études andines [En ligne], 43 (2) | 2014, mis en ligne le 08 août 2014, consulté le 06 novembre 2020. URL : http://journals.openedition.org/bifea/5257 ; DOI : https:// doi.org/10.4000/bifea.5257

\section{(ब) $\odot \Theta$}

Les contenus du Bulletin de l'Institut français d'études andines sont mis à disposition selon les termes de la licence Creative Commons Attribution - Pas d'Utilisation Commerciale - Pas de Modification 4.0 International. 


\title{
Quespillo et ses compagnons : la figure du gracioso dans le théâtre quechua colonial*
}

\author{
Rossella Martin**
}

\section{Résumé}

Personnage-type incontournable du théâtre du Siècle d'Or espagnol, le gracioso figure dans toutes les pièces connues du répertoire dramatique en langue quechua du Cuzco colonial. Écrits par des curés, ces drames étaient destinés à être représentés devant les élites indigènes, qui en étaient vraisemblablement les commanditaires.

Cet article se propose de contribuer à la réflexion pluridisciplinaire sur les processus de reconstruction identitaire de la population autochtone à l'époque coloniale, en s'intéressant aux modalités de cette opération de transfert culturel et à ses acteurs qui, en s'appropriant la figure du gracioso, élaborèrent une image susceptible de faire sens dans I'univers symbolique andin.

Mots-clés : gracioso, théâtre quechua colonial, transfert culturel, construction identitaire indigène

\section{Quespillo y sus compañeros: la figura del gracioso en el teatro quechua colonial}

\section{Resumen}

Personaje-tipo indispensable del teatro del Siglo de Oro español, el gracioso figura en todas las piezas conocidas del repertorio dramático en lengua quechua del Cuzco colonial. Estos dramas, escritos por sacerdotes, estaban destinados a ser representados frente a las élites indígenas, quienes eran probablemente los patrocinadores.

* Une première version de cet article a été présentée dans le symposium Entre conversión, inversión y subversión : los textos en lenguas amerindias escritos por autores indígenas en la época colonial, coordonné par Sabine Dedenbach-Salazar dans le cadre du 54 ICA (Vienne, 15-20 juillet 2012).

** Chercheure post-doctorante, Institut national de Langues et Civilisations orientales (Inalco). E-mail : rossella.martin@wanadoo.fr 
Este artículo propone contribuir a la reflexión pluridisciplinaria sobre los procesos de reconstrucción identitaria de la población indígena durante la época colonial, centrándose particularmente en las modalidades de esta operación de transferencia cultural y en sus actores, que se apropiaron de la figura del gracioso para elaborar una imagen que tuviera sentido en el universo simbólico andino.

Palabras clave: gracioso, teatro quechua colonial, transferencia cultural, construcción identitaria indígena

\title{
Quespillo and his companions: the character of the gracioso in the Quechua Colonial Theatre
}

\begin{abstract}
Essential character-type of the Spanish theatre during the Golden Age, the gracioso can be found in all known pieces of dramatic repertoire in Quechua language of the colonial Cuzco. These dramas, written by priests, were destined to be shown in front of the indigenous elite, who were probably the sponsors. This article contributes to the multidisciplinary thought that concentrates on the reconstruction process of native identity during the colonial era, focusing on the methods of this cultural transfer and its actors who, using the gracioso figure, developed an image which was able to have a significance in the symbolic universe of the Andes.
\end{abstract}

Keywords: gracioso, quechua colonial theatre, cultural transfer, native identity construction

\section{INTRODUCTION}

La production théâtrale coloniale en langue quechua naît et se développe à Cuzco au cours des XVII e et XVIII e siècles. II s'agit d'une production savante qui s'adresse à un public urbain, formé - même si à un degré variable — à la culture occidentale et familier des drames du théâtre du Siècle d'Or espagnol, qui constituent les modèles littéraires de cette production. Le corpus du théâtre quechua colonial comprend actuellement six oeuvres. Les plus anciennes sont deux autos sacramentales écrits vers le milieu du XVII siècle par le prédicateur et écrivain cuzquénien Juan de Espinosa Medrano : El robo de Proserpina y sueño de Endimión et El hijo pródigo'. Suivent trois « comedias marianas »², El pobre más rico, composée vers la fin du XVIII ou le début du XVIII siècle par le prêtre Gabriel Centeno de Osma, et les anonymes Usca Paucar et El milagro del Rosario, qui

1 El robo de Proserpina y sueño de Endimión fut vraisemblablement écrit vers la fin de la décennie 1640, à l'époque où Espinosa était séminariste au collège de San Antonio Abad (Itier, 2010 : 1012). Plus tardif, El hijo pródigo pourrait avoir été composé en 1668, à l'occasion des célébrations qui s'organisèrent à Cuzco pour la visite du vice-roi comte de Lemos (César Itier, communication personnelle).

2 J'emprunte l'expression « comedias marianas » à Carlos García-Bedoya, qui désigne ainsi El pobre más rico et l'Usca Paucar car ces drames sont liés à des sanctuaires mariaux et traitent le thème du salut par l'intercession de la Vierge (García-Bedoya, 1998 : 333). 
datent du milieu du XVIII e siècle. Nous avons enfin la célèbre comedia d'argument pseudo-historique Ollantay, composée en 1782 par le curé Antonio Valdez³.

À l'exception peut-être de El milagro del Rosario, oeuvre moins bien écrite et qui intègre de nombreux éléments de la tradition orale, ces pièces montrent une excellente maîtrise des codes littéraires du théâtre baroque espagnol : versification poly-métrique avec prédominance des octosyllabes et maniement de la rime — même si avec de nombreuses irrégularités - dans toutes les pièces ; division en trois actes avec insertion de fragments lyriques et présence de personnages-types comme le galán et le gracioso dans les comedias ; action renfermée en un seul acte, présence de personnages allégoriques et thématique eucharistique dans les autos sacramentales.

Espinosa, Centeno et Valdez étaient des ecclésiastiques ; il est fort probable que les auteurs des anonymes Usca Paucar et El milagro del Rosario l'étaient eux aussi. Dans le Cuzco colonial, où l'Église détenait le monopole de la culture et où l'éducation des fils des caciques et de la noblesse d'ascendance inca était confiée aux Jésuites (Alaperrine-Bouyer, 2007), les religieux formaient une classe d'intellectuels bilingues, étroitement liée aux groupes seigneuriaux indigènes. II n'était pas rare d'ailleurs que les membres du clergé et des ordres réguliers fussent apparentés aux élites autochtones, dont ils soutenaient les points de vue et les aspirations socio-politiques (García-Bedoya, 2000 : 163). Juan d'Espinosa Medrano (né en 1628 ou en 1630 et mort en 1688) semble avoir été un métis de mère indienne ${ }^{4}$; on ignore I'origine ethnique de Gabriel Centeno de Osma ainsi que les dates de sa naissance et de sa mort (Meneses, 1983 : 378) ; quant à Antonio Valdez (né vers 1732-1740 et mort en 1816), ses biographes hésitent entre l'identité métisse et créole (Meneses, 1977 ; 1983 ; Porras, 1954a ; b ; 1955). Comme au Pérou I'accès aux ordres religieux resta interdit aux Indiens et, à quelques exceptions près, aux Métis jusqu'au milieu du XVIIIe siècle, on peut exclure que les auteurs

3 La chronologie du théâtre quechua que je propose, est celle présentée par César Itier dans le cadre du cours de littérature quechua dispensé à I'Inalco. À l'exception de El robo de Proserpina, dont les manuscrits et le texte en translittération moderne ont été publiés (Itier, 2010), et d'Ollantay, dont j'ai fait moi-même la translittération à partir du manuscrit Justiniani, les autres textes du théâtre quechua sur lesquels se base cette étude, m’ont généreusement été fournis par César Itier en version translittérée. C'est moi qui ai traduit en français tous les extraits rapportés dans l'article. César Itier les a revus et corrigés. Teodoro Meneses a publié la traduction espagnole de tous les textes (Meneses, 1983) à l'exception de El milagro del Rosario, dont le manuscrit a été découvert par César Itier dans le fond Markham de la British Library.

4 L'identité ethnique d'Espinosa Medrano est très controversée. D'après Luis Jaime Cisneros et Pedro Guibovich Pérez, qui ont essayé d'établir la biographie d'Espinosa, la « légende » de l'origine métisse s'appuierait sur de simples conjectures et manquerait d'une base documentaire fiable (Cisneros \& Guibovich, 1988 : 331-335). Certains indices semblent cependant suggérer le contraire. Dans le prologue au recueil de sermons La novena maravilla (paru en édition posthume en 1695), son biographe et ancien disciple Agustín Cortés de La Cruz écrit qu'Espinosa était « hijo de sus obras » (Cisneros \& Guibovich, 1988 : 334). Or, comme le rappelle Rodríguez Garrido, cette expression était régulièrement employée pour désigner un homme dont les origines étaient obscures - soit qu'il ne fût pas de sangre limpia soit qu'il fût un enfant naturel ou illégitime -, et qui ne devait sa renommée qu'à la qualité de ses œuvres (Rodríguez Garrido, 1994 : 32). Aussi ne trouve-t-on dans son testament aucune référence à ses origine et filiation, ce qui était inhabituel dans ce type de documents qui commençaient généralement par une description généalogique (Vitulli, 2011 :15). 
des drames quechuas coloniaux aient été d'origine autochtone. En tout cas, d'un point de vue socio-culturel, ils appartenaient au groupe créole et avaient reçu une solide formation dans des institutions d'éducation supérieure comme le collège séminaire de San Antonio Abad à Cuzco, où Espinosa et Valdez firent leurs études (Cisneros \& Guibovich, 1988 : 336-337 ; Meneses, 1977 : 52).

Le fait que ces drames soient écrits dans une langue amérindienne ne doit surtout pas être interprété comme le signe de l'identité ethnique indigène de leurs auteurs. Une variante dialectale de la langue quechua avait été utilisée par les Incas comme langue véhiculaire de leur empire. Grâce à sa diffusion importante en tant que langue de communication interethnique et au prestige dû à son statut de « lengua cortesana » ou langue de la cour inca de Cuzco, le quechua avait été choisi par le Troisième Concile de Lima (1582-1583) pour servir les besoins de l'évangélisation. Un abondant corpus, comprenant des manuels de catéchèse, des grammaires et des dictionnaires, avait rapidement vu le jour. Le quechua était ainsi devenu la «lengua general » la plus diffusée de la vice-royauté et, dans le Cuzco des XVII et XVIII siècles — dont la population était à dominante indienne - , toutes les composantes ethniques de la société, y compris les Créoles, le comprenaient et le parlaient avec fluidité. Seuls les Espagnols venus de la métropole — ceux qui étaient appelés avec mépris « chapetones » — ne savaient pas s'exprimer en quechua.

Or, bien que la langue dans laquelle nos drames ont été composés soit un idiome élaboré, fruit d'un projet cultivé et puriste (Lienhard, 1992 : 161), riche en archaïsmes lexicaux — mots sortis de l'usage — et en archaïsmes sémantiques — mots utilisés en un sens sorti de l'usage — - elle restait compréhensible pour la population urbaine de Cuzco et, notamment, pour l'élite indigène qui constituait le destinataire privilégié de ce théâtre.

En fait, comme le souligne Carlos García-Bedoya, le théâtre quechua colonial «no se define por la identidad étnica de sus productores » mais plutôt « por su relación orgánica con un grupo social, el de las noblezas indígenas coloniales, quienes eran tanto los patrocinadores como los consumidores de esta producción simbólica » (García-Bedoya, 1998 : 326). Même s'il n'existe aucun document qui permette de confirmer cette affirmation - qui doit par conséquent être considérée comme une simple hypothèse - , elle me paraît cependant très convaincante. Il est en effet vraisemblable que l'élite indigène de Cuzco, étant privée de l'accès aux ordres religieux et ne disposant pas d'une production littéraire propre, déléguait à des ecclésiastiques créoles la tâche de la représenter en écrivant des drames dont les protagonistes étaient des nobles incas. II ne faut pas oublier que dans I'ancienne capitale du Tahuantinsuyo se trouvait « la mayor concentración de linajes nobiliarios indígenas, orgullosos de su pasado y su tradición » (Flores Espinoza, 2001 : 22). À partir du milieu du XVII e et tout au long du XVIII e siècle, les revendications identitaires et politiques de ce groupe social s'exprimèrent, entre autres, à travers une appropriation créative de l'image de I'Inca et des symboles qui lui étaient liés. Ce phénomène, connu sous le nom de «Renaissance inca »5, bénéficia du

5 Parmi les nombreux travaux sur la Renaissance inca, il faut au moins citer l'étude pionnière de John Rowe «El movimiento nacional inca del siglo XVIII » (Rowe, 1954 : 17-47). 
concours des arts plastiques et décoratifs et des arts dits « éphémères 》 — défilés de rue et représentations théâtrales essentiellement. Les descendants des anciens Incas participaient aux festivités civiles et religieuses en arborant les vêtements traditionnels et les insignes de la royauté inca, la mascapaycha et un médaillon en forme de disque solaire sur la poitrine6. Ainsi habillés, ils posaient pour des portraits qui ornaient les murs de leurs maisons; ils s'entouraient d'objets précolombiens comme les gobelets en bois appelés queros ${ }^{7}$ — qu'ils utilisaient toujours en couple comme le faisaient les anciens Incas - et se transmettaient des légendes relatives à la geste épique de leurs ancêtres — légendes dont est vraisemblablement issu Ollantay (Martin, 2007). Tout en leur fournissant I'occasion de mettre en scène leur passé glorieux, le théâtre quechua leur permettait de s'attribuer une place symbolique importante dans l'espace public de la cité. García-Bedoya insiste sur le fait que cette élite andine disposait non seulement des moyens de parrainer des cérémonies publiques mais aussi d'exercer un mécénat artistique, en promouvant notamment les peintures de la Escuela cuzqueña et la production dramatique en langue quechua (García-Bedoya, 2000 : 196-198).

De la même manière, nous pouvons supposer que les acteurs qui interprétaient les pièces du théâtre quechua appartenaient aux familles de l'élite indigène. II s'agissait probablement d'étudiants ou d'anciens étudiants du collège San Borja de Cuzco, régenté par les Jésuites. Seulement des acteurs ayant reçu une certaine instruction, en effet, pouvaient assimiler des textes écrits dans un idiome qui - comme nous venons de le rappeler - se caractérisait par des tournures et des mots savants, inusités dans la langue de tous les jours et, par conséquent, difficiles à retenir et à apprendre par cour.

Malheureusement, nous ne possédons aucun témoignage écrit qui nous renseigne sur les conditions de représentation de ce théâtre. Et ce ne sont certainement pas les rares didascalies de nos drames qui peuvent nous fournir des informations supplémentaires. On sait qu'en Espagne comme en Amérique, les autos sacramentales étaient représentés sur les places publiques à l'occasion de la fête du Corpus Christi. Les acteurs jouaient sur des tréteaux ou sur des chars mobiles surélevés qui avaient préalablement défilé au milieu de la foule en liesse. On sait également qu'existait à Cuzco un corral de comedias mais rien ne nous permet d'affirmer que nos drames y furent jamais mis en scène. García-Bedoya émet la double hypothèse que les représentations du théâtre quechua avaient lieu dans le collège San Borja de Cuzco et dans les maisons de l'élite indigène, devant un public restreint (García-Bedoya, 2000 : 197). Cependant, le manuscrit qui nous a transmis El milagro del Rosario est une copie réalisée par un curé en 1793,

6 La maskapaycha était une frange de laine rouge qui descendait sur le front du souverain inca, dont la tête était ceinte par une tresse multicolore, le llawt'u. Quant au pendentif figurant le soleil, qui apparaît sur la poitrine des Incas représentés dans les tableaux de l'École de Cuzco, il s'agit d'une création iconographique de l'époque coloniale, venue probablement remplacer les grands ornements d'oreilles qui valurent aux anciens Incas le surnom de « orejones » et qui n'étaient plus d'usage parmi leurs descendants.

7 Du quechua qiru « bois ». 
pour une mise en scène qui devait se dérouler dans le village d'Acos le jour de la fête du Rosaire8. II est possible qu'Ollantay ait été représenté dans le quartier révolutionnaire d'Azangaro, à l'époque où Valdez se trouvait auprès de Diego Cristóbal Tupac Amaru - le cousin germain de José Gabriel Tupac Amaru et son successeur à la tête de la Grande Rébellion de 1780-1783 —, pour le persuader d'accepter les conditions de la reddition imposées par les autorités coloniales (Itier, 2006).

Ce qui, en dépit de l'absence de toute documentation, me porte à croire que les drames du théâtre quechua étaient représentés devant un public assez large et hétérogène. C'est aussi ce qu'invite à penser le fait que le personnage du gracioso s'adresse fréquemment aux femmes - qu'il prend pour cible de ses railleries - et à différentes catégories socio-ethniques de spectateurs, comme dans cet extrait de El pobre más rico :

\section{Qispillu}

Nuqapas hinallataqmi kusikuspapas

anchataqmi llakini qamkunakta haqispa.

Diosllawanchik tayta llama michiq

ansi manaraqchu waqankichik?

Diosllawan k'usilluhina qara ñawi payakuna,

Diosllawanchik sayaq rinri sipaskuna,

Diosllawan hawas phuspuhina

qara unku mistikuna,

Diosllawanchik titi corpiño mistisakuna,

mankap sikinman waskha sinrisqahina

chukchayuq warmikuna, Diosllawan.

\section{Quespillo}

Moi aussi, bien que je sois de nature joyeuse,

je souffre beaucoup en vous quittant.

Adieu messieurs les pasteurs de lamas, ne pleurez-vous pas encore?

Adieu vieilles femmes aux paupières de singes,

adieu jeunes filles aux oreilles bien droites,

adieu métis aux chemises raides comme des fèves grillées, adieu métisses aux corsages de plomb,

adieu femmes à la chevelure (tressée) comme

les cordes attachées aux extremités de la marmite, adieu !

El pobre más rico, vv. 1106-1117

Et c'est justement au personnage du gracioso que nous allons nous intéresser dans les pages qui suivent. Ce type théâtral constitue en effet un exemple de transfert culturel parfaitement réussi. Et il n'est pas besoin de rappeler que toute opération

8 «Soy de D(on) Mariano Perez y Vertiz / Cordinado en el mes de Agosto de 1793 / para el Dia del Rosario en Acos / y ne me bendo ni me doy el sujeto q(u)e fuere Ynteresado de mi costara su pla / ta y trabajo Como p(ar)a mi y para fee / firmó mi dueño ». 
de transfert culturel intervient dans un contexte bien précis, pour servir les intérêts d'un certain type d'acteurs sociaux qui l'utilisent à des fins spécifiques.

Or, si la figure du noble inca, protagoniste de nos drames, peut être considérée - pour des raisons sur lesquelles nous aurons l'occasion de revenir — comme une sorte de miroir qui reflète une image flatteuse de l'élite indigène, comment expliquer l'importance du gracioso, qui est présent dans tous les drames de notre corpus et qui joue même un rôle de co-protagoniste dans les comedias marianas du XVIII siècle ? La verve comique du personnage est-elle suffisante pour justifier son succès auprès du public cuzquénien ? Qui représentait-t-il dans l'imaginaire local ? À quelles attentes répondait-il ? Et de quels messages se faisait-il porteur?

Pour en savoir un peu plus, nous allons examiner dans un premier temps les caractéristiques du gracioso andin, aussi bien celles qu'il a héritées du modèle espagnol que celles qu'il a acquises au contact de la culture locale. II sera temps alors, pour le second moment de l'enquête, de suivre le processus de transformation que le personnage subit dans le passage des autos sacramentales du XVII siècle aux comedias du XVIII . Ce qui va nous permettre d'apporter quelques réponses à nos questions et d'étayer I'hypothèse selon laquelle le gracioso andin était chargé de transmettre des valeurs qui, non seulement correspondaient aux orientations didactiques de ce théâtre, mais satisfaisaient aussi les attentes et le besoin de reconnaissance sociale du public populaire, qui assistait de plus en plus nombreux aux représentations.

\section{LE GRACIOSO. UN TRANSFERT CULTUREL RÉUSSI}

Si Lope de Vega semble avoir été le premier à avoir introduit le gracioso ou «figura del donaire » dans le théâtre espagnol, et plus exactement dans la comedia La francesilla datée de 1596 (Gómez, 2006 : 11), les origines du personnage remontent loin dans le temps. On s'accorde à lui donner pour ancêtre le servus currens du théâtre de Plaute, l'esclave intrigant et astucieux qui met son expérience en ruses et en tromperies au service de l'adulescens et de ses conquêtes amoureuses. Plus proches de l'époque de Lope, nous trouvons il servo de la comédie érudite italienne de la Renaissance et l'Arlecchino de la très populaire Commedia dell'Arte (Dumas, 2004 : 46). Et il ne faut pas oublier la parenté du gracioso avec la figure contemporaine du pícaro, dont le représentant le plus célèbre est le protagoniste de El Lazarillo de Tormes (anonyme, 1554), de même que sa dette vis-à-vis du pasteur «bobo » des églogues du XV siècle de Juan del Encina et du « simple » des pasos du XVI e siècle de Lope de Rueda (Gómez, 2006 : 19).

\section{1. Agent du rire : les attributs comiques du gracioso}

Ce riche héritage culturel est à l'origine de la complexité du gracioso, figure située à mi-chemin entre le type codifié et le personnage individualisé, et de la plasticité dont il fait preuve dans le passage d'une pièce à une autre. Cela explique aussi 
que le comique dont le gracioso est l'agent, tient d'une part de ce que l'on appelle couramment le « comique volontaire », lorsque le personnage fait de l'humour dans l'intention précise de divertir le public grâce, par exemple, à ses traits d'esprit et à ses jeux de mots et, d'autre part, du « comique involontaire », lorsque le personnage suscite I'hilarité à cause de ses bévues, de sa maladresse ou en tant que victime des circonstances. Ces deux types de comique sont réunis dans une scène de I'Usca Paucar, où le pauvre Quespillo est roué de coups par quatre diables qui veulent l'obliger à révéler où est caché son maître (Usca Paucar, vv. 1659-1686).

Si le comique de la scène est assuré par les plaisanteries de Quespillo — qui ne renonce pas à faire des blagues même sous une pluie de bastonnades -, il repose également sur le fait que le gracioso devient le bouc émissaire de la situation, les diables se défoulant sur lui faute de pouvoir attraper son maître. Que son humour soit assumé ou subi, la fonction principale du personnage est celle de faire rire et d'amuser les spectateurs, en allégeant ainsi la tension dramatique de la pièce.

Dans le théâtre baroque espagnol, le nom du gracioso témoigne le plus souvent de son origine paysanne, et cela en opposition avec le nom du galán qui indique son appartenance à la classe nobiliaire. Dans le théâtre quechua colonial, tout en « s'andinisant », le nom du gracioso conserve son caractère populaire, de même qu'une coloration comique qui peut aller jusqu'au sobriquet ridicule. Le gracioso de El robo de Proserpina s'appelle Taparaco, le serviteur du dieu des Enfers, Plutón. De toutes les dramatis personae de cet auto sacramental dans lequel les personnages allégoriques se doublent de figures mythologiques, il est le seul à porter un nom lié à l'univers andin, un taparaku étant un papillon nocturne censé être de mauvais augure9.

Dans El hijo pródigo - qui est une transposition andine de la célèbre parabole évangélique - le gracioso est Ukhu, le « Corps ». Ce nom fait référence à I'un des traits constitutifs du personnage, sa sujétion aux appétits charnels, et rappelle I'opposition fondamentale corps-esprit qui caractérise la relation entre le gracioso et son maître, en l'occurrence Cristiano, personnification de l'âme.

La figure du serviteur dans les trois comedias marianas, El pobre más rico, Usca Paucar et El milagro del Rosario — dont les deux dernières peuvent être considérées comme des remakes littéraires de la première — s'appelle Quespillo (Qispillu). Ce nom est formé du suffixe espagnol -illo, un diminutif qui entre dans la composition onomastique de plusieurs graciosos du théâtre du Siècle d'Or espagnol, tels que Rodriguillo dans la Ingratitud vengada (1590-1595) ou Hernandillo dans La ocasión perdida (1599-1603) de Lope. Il est précédé par la racine verbale quechua qispi-,

9 Je dois cependant signaler que César Itier démontre que le nom original du gracioso de El robo de Proserpina était Ascálafo. Ce nom était celui du personnage transformé en hibou par Cérès dans les Métamorphoses d'Ovide, qui constituent une des sources littéraires de la pièce d'Espinosa. Le nom d'Ascálafo aurait été remplacé par celui de Taparaco par un copiste tardif, soucieux de changer — peut-être en vue d'une représentation — « este nombre exótico y oscuro por un término quechua susceptible de tener resonancias en la cultura del público » (Itier, 2010 : 38). 
dont une des significations est celle de « se sauver, s'enfuir » en référence à une autre caractéristique du personnage, sa couardise.

Enfin, le gracioso d'Ollantay s'appelle Piqui Chaqui (Piki Chaki), «Pied-de-Puce» (de piki « puce » et chaki « pied »), nom qui évoque le caractère « pouilleux » du personnage, les puces étant des parasites qui se logent sur le corps et, notamment, dans les extrémités des pauvres qui marchent à pieds nus.

Il est fort dommage que les didascalies de nos drames ne fournissent pas d'indications sur l'apparence physique du gracioso. Si Taparaco entre en scène habillé comme un hortelano (El robo de Proserpina, didascalie v. 535), car le serviteur de Plutón est également le jardinier des Enfers, aucune didascalie ne décrit Ukhu, le gracioso de El hijo pródigo. Au cours de la pièce on s'adresse cependant à lui en I'appelant wiksasapa " gros ventre» (v. 257) et wiksa q'ipi « ventre en sac» (v. 735), ce qui pourrait signifier qu'à l'instar du type du glouton dans l'iconographie médiévale, le personnage était représenté avec un gros ventre. Quant à nos trois Quespillo, les didascalies qui les introduisent les décrivent comme d'aspect muy roto (El pobre más rico, didascalie v. 138) et ridículo (Usca Paucar, didascalie initiale; El milagro del rosario, didascalie $\mathrm{v}$. 136). Ce qui nous permet de supposer qu'au ridicule vestimentaire du gracioso, s'associait une certaine difformité physique destinée à susciter le rire par contraste avec l'élégance et la prestance du galán.

\section{2. Galán-gracioso : une relation de complémentarité antithétique}

Ce qui caractérise en effet le gracioso par rapport aux autres figures de serviteur du théâtre espagnol, est la relation de complémentarité qu'il entretient avec son maître, dont il est le faire-valoir. Alors que le galán véhicule les valeurs de la noblesse, son sens de l'honneur, ses goûts raffinés et ses penchants spirituels, le gracioso révèle sa basse extraction sociale en se faisant le porte-parole de préoccupations beaucoup plus terre-à-terre, son premier souci étant celui de manger à sa faim. Cette antithèse est magistralement illustrée par le couple Cristiano-Ukhu dans El hijo pródigo. Le véritable culte que le gracioso voue à la nourriture et à la boisson, le porte à élire comme interlocuteurs privilégiés son ventre et son gosier : Ay, wiksallay! Ay, tunqurllay! / Qullqi kaptinchu q'aymarayana? / Qirutañam musphapukuni. «Ah, mon ventre chéri ! Ah, mon cher gosier ! / S'il y avait de l'argent, est-ce que vous seriez à sec ? / Je rêve à des gobelets en bois (pleins de vin)!» (El hijo pródigo, vv. 159-161). Et si Cristiano ose lui reprocher sa gourmandise, Ukhu se lance dans une évocation lyrique et passionnée de tous les types d'aliments andins (El hijo pródigo, vv. 266-281).

Gouverné par ses appétits charnels, le gracioso est attiré par les femmes qu'il voudrait « consommer», comme s'il s'agissait de nourriture. Ainsi, en présence de la coya Aycha, «la reine Chair », le premier réflexe d’Ukhu est de chercher à la « goûter» en lui mordant la main : 


\section{Aycha}

Manaraqchu riqsiwanki?

Nuqaqa hatun qhapaq quyam

munay, maskay, Aycham kani.

\section{Ukhu}

Kaychus Aycha? Wararáy!

Hankutapas mikhuymanmi !

[...]

\section{Aycha}

Makiytaqa much'aykuypas, anchatam kusichiwanki chika qhapaq kaspa quncha.

\section{Ukhu}

P'utuyp'utuy makiykitari kaniptiy phiñakunkichus, tumpalla k'utuykusqayki.

\section{La Chair}

Ne me connais-tu pas encore? Je suis une reine grande et puissante, aimée et convoitée, je suis la Chair.

\section{Le Corps}

C'est ça la Chair ? Qu'elle est belle!

Je pourrais la manger toute crue! [...]

\section{La Chair}

Tu peux baiser ma main car je suis très contente de savoir que tu es riche, neveu.

\section{Le Corps}

Est-ce que ça t'embête si je mords ta main grassouillette?

Je vais juste la grignoter un peu...

El hijo pródigo, vv. 366-370; 438 -443

En se penchant sur la main de la courtisane Aycha pour la mordre, Ukhu imite de façon parodique I'hommage du baise main fait par le galán à la dama. Le sentiment amoureux chez le gracioso s'exprime en effet à travers des mots et des actions qui constituent, la plupart du temps, une transposition burlesque de ceux du galán.

Il arrive que la comedia mette en scène, parallèlement aux amours du galán et de la dama, les amours du gracioso et de la criada, juxtaposition qui provoque des effets de comique par contraste. C'est le cas dans El pobre más rico, où Quespillo cherche à gagner les faveurs de la graciosa Achira. Mais alors que le galán et la dama se promettent un amour absolu et éternel, le gracioso et la criada partagent une conception de l'amour beaucoup plus relative et éphémère. Ainsi, lorsque sa maîtresse critique sévèrement les femmes qui aiment plusieurs hommes à la fois, la réponse pragmatique d'Achira ne se fait pas attendre :

\section{Quri Umiña}

Ima sunqum kanman, ñiway, munasqanchikta wasanchaq? Iskay kimsa munaq warmi

munasqanta wasanchakuq manapuniraqmi yachanchu imam munakuypas ñispa.

\section{Cori Omiña}

Dis-moi, quel coeur pourrait trahir son aimé ?

La femme qui aime deux ou trois (hommes) à la fois, celle qui trahit son aimé, ignore complètement ce qu'est l'amour. 


\section{Achira}

Niway ari kunan quya

huk hatun mayukta chimpachwan

huk waskhallamanta hap'ikuspa.

Chay p'itiptinri manachu

p'unquman urmaykuchwan?

Tawa pichqa waskha kaptinri

huk p'itinptinpas wakinqa

hap'ikunapaq kanmanmi.

Kay munakuypa sinchi mayunta

hukllakta hap'ikuspa chimpasun.

P'itikuptin urmaykusun

waqaq llakikuypa p’unqunman.

El pobre más rico, vv. 1942-1959

\section{Achira}

Maintenant répond-moi, ma reine :

si nous traversons un grand fleuve en nous attachant à une seule corde,

n'est-ce pas vrai que si cette corde lâche,

nous allons tomber dans le

courant?

Mais si les cordes sont quatre ou cinq,

même si l'une d'entre elles lâche, les autres

vont nous soutenir.

Lorsque nous traversons le puissant fleuve de

l'amour attachées à une seule corde,

si elle se détache nous allons tomber

en pleurant dans le gouffre du malheur.

Le comique par contraste des amours parallèles galán-dama et gracioso-criada repose aussi sur le lexique du langage amoureux. Alors que le couple de nobles s'exprime selon les codes de l'éloquence galante néo-pétrarquiste, le langage des serviteurs est souvent trivial, leurs échanges verbaux pouvant aller jusqu'aux insultes en rafale (El pobre más rico, vv. 2158-66).

D'autre part, il est intéressant de remarquer que les dialogues entre le gracioso et la criada sont parmi les plus savoureusement andins du théâtre quechua. C'est le cas, par exemple, lorsque Quespillo et Achira réactivent la métaphore du chat et de la souris, très fréquemment employée par la tradition orale :

\section{Qispillu}

Yachakuy, huk'ucha, manam qispinkichu.

Manapunich ñuqa Qispilluchu kayman

qam sitki p’asñakta qispichiqqa manach.

\section{Achira}

Yuyaytaq, misitu, « hap'isaqcha » ñispa,

\section{Quespillo}

Sache-le, petite souris : tu ne te sauveras pas!

Moi, Quespillo, je ne pourrais jamais

te laisser t'en sortir, petite sauvageonne. Jamais!

\section{Achira}

Pense, chaton, lorsque tu dis que tu vas m'attraper, 
Qispillu kaspapas manapuniraqcha

chayray qispipiqa qhawaykukuwaqchu. que même en étant Quespillo, jamais tu ne pourras te regarder dans ce miroir !10

El pobre más rico, vv. 2143-2148.

Présence démystifiante dans la pièce, le gracioso tourne en raillerie l'idéalisme amoureux de son maître. En qualité de dépositaire des secrets du coeur du galán, il reçoit ses confidences amoureuses, comme dans la scène d'exposition d'Ollantay. Le rôle de confident, joué par le gracioso, remplit ici une fonction très importante car il permet aux spectateurs d'obtenir tous les éléments nécessaires à la compréhension de la situation initiale, sans que l'auteur doive avoir recours au monologue du protagoniste. Dans la comedia espagnole, le gracioso s'efforce généralement de freiner l'ardeur de son maître, en l'invitant à la prudence et en lui prodiguant des conseils avisés. C'est ainsi que Piqui Chaqui met en garde Ollantay contre le danger que représente sa liaison avec la fille de l'Inca, et lui rappelle que le monde ne manque pas de jolies femmes vers lesquelles il pourrait se tourner sans risquer sa vie :

\section{Ullantay}

Pikichaki, rikunkichu

Kusiquyllurta wasinpi ?

\section{Piki Chaki}

Ama inti munachunchu chayman churakunaytaqa!

Manachu qamqa manchanki Inkap ususin kasqanta?

\section{Ullantay}

Chaypas kachun, munasaqmi

chay lulukusqay urpita.

Nam kay sunquy paypaq chita,

payllallatam munachkani.

\section{Piki Chaki}

Supaychá raykusqasunki,

icha qamqa musphankipas?

Hinantinpim warma sipas

\section{Ollantay}

Piqui Chaqui, as-tu vu

Cusi Coyllur dans son palais?

\section{Piqui Chaqui}

Que le Soleil ne permette pas

que je me mette dans un tel pétrin!

Le fait qu'elle soit la fille de l'Inca ne te fait pas peur?

\section{Ollantay}

Quoi qu'il advienne, j'aimerai (toujours)

cette tendre colombe !

Désormais mon coeur lui appartient, il n'y a qu'elle que j'aime !

\section{Piqui Chaqui}

Est-ce que c'est le diable qui t'incite à pécher ou tu es en train de délirer? Partout il y a des jeunes filles (à aimer),

${ }^{10}$ Le nom du gracioso sert ici un double jeu de mots humoristique : employé d'abord dans sa signification de « se sauver » d'après la racine verbale qispi-, il prend ensuite le sens de « miroir » d'après le substantif qispi, dérivé de la racine verbale qispimu- " passer à travers », qui désigne une surface transparente ou du verre. 


\author{
anchatam rukupakunki! \\ Ima p'unchawpi yachanqa \\ Inka yuyaykusqaykita \\ qhuruchinqan umaykita, \\ qamtaq kanki aycha kanka! \\ Ollantay, vv. 1-18 (in Valdez, 1938)
}

avant que tu ne sois trop vieux !

Le jour où I'Inca connaîtra

tes pensées,

il te fera couper la tête

et te transformera en viande

rôtie!

Ce serait donc se faire du gracioso une idée erronée que de voir en lui seulement un bouffon insolent et farceur, car ce représentant de la classe populaire incarne également la sagesse populaire. Son bon sens et son esprit pratique lui permettent d'échapper à maints dangers et de sortir le galán de situations très difficiles. Plein de ressources et capable de se débrouiller en toutes circonstances, le gracioso est un compagnon d'épreuves dévoué qui peut partager avec son maître les mêmes risques et les mêmes revers de fortune. Nous allons voir maintenant que ces traits positifs du personnage vont s'accentuer dans les comedias marianas du XVIII siècle, en faisant du gracioso une figure riche et complexe à même de rivaliser avec celle du galán.

\section{DE TAPARACO À PIQUI CHAQUI : ÉVOLUTION DU PERSONNAGE DU GRACIOSO}

Nous avons vu que le principal trait distinctif du gracioso par rapport aux autres personnages de serviteur du théâtre baroque espagnol, est le rapport de complémentarité antithétique qu'il entretient avec le galán. En réalité, le gracioso n'est pas seulement le contrepoint comique, la « doublure contrefaite » (Dumas, 2004 : 58), I'alter-ego du galán ; il est aussi son subordonné hiérarchique aussi bien au sein de l'action dramatique, en tant que domestique à son service, que dans le système actanciel de la comedia, le gracioso ne pouvant pas exister sans le galán ou, autrement dit, ne pouvant pas remplir le rôle de protagoniste. C'est pourquoi, avant de s'intéresser à l'évolution du gracioso dans le théâtre quechua colonial, il convient de dire quelques mots à propos des changements qui interviennent dans le traitement du personnage du noble inca, héros de ce théâtre.

\section{1. La figure du noble inca dans le théâtre quechua colonial 11}

Le protagoniste de la comedia baroque espagnole est, par convention, un noble ou un personnage de haut rang. Dans le Cuzco colonial, où l'élite indigène constituait vraisemblablement — comme nous l'avons rappelé en introduction —

11 Je renvoie aux études de Meneses (Meneses, 1983), Burga (Burga, 1988 : 314-318), Mannheim (Mannheim, 1990 : 139-178), Chang-Rodríguez (Chang-Rodríguez, 1991 : 209-245), Itier (Itier, 1995 : 89-106) et García-Bedoya (García-Bedoya, 1998 : 325-338 ; 2000 : 203-206), pour une analyse plus détaillée de la figure du noble inca dans le théâtre quechua colonial. 
le destinataire et, en même temps, le commanditaire du théâtre quechua, le protagoniste est un noble inca.

Or, si nous prenons les deux autos sacramentales écrits par Espinosa au XVII siècle, nous y rencontrons effectivement des personnages d'Inca. Cependant, force est de constater que leur caractérisation est loin d'être positive. Dans El robo de Proserpina, I'héroïne éponyme (l'Âme) est partagée entre le berger Endimión (le Christ) et le roi des Enfers, Plutón (Lucifer). La didascalie qui introduit ce dernier personnage, le présente « vestido de una camiseta colorada, muy rica, y un manto negro con vueltas verdes, todo él salpicado con estrellas de oro, y una guirnalda en lugar de mascapaicha ». Le roi des Enfers est accompagné par quatre Manes «vestidos de camisetas igualmente salpicadas de estrellas » (Itier, 2010 : 56). Comme I'explique Itier dans une note à son édition de El robo de Proserpina, la fonction de la mascapaycha qui orne la tête de Plutón, est essentiellement celle d'indicateur culturellement reconnaissable de la condition royale du personnage, exactement comme les camisetas qui évoquent I'unku ou tunique inca (Itier, 2010 : note p. 57). Mais même si, en représentant le Diable sous les traits de I'Inca, Espinosa se préoccupe d'abord de fournir à son public une image familière et vraisemblable de la royauté, il n'en reste pas moins que l'accumulation de ces indices culturels 12 finit par superposer à la figure mythico-allégorique de PlutónLucifer celle historique de I'Inca.

Dans El hijo pródigo, la figure de I'Inca est incarnée par le personnage de Mundo, à la cour duquel se rend Cristiano, fourvoyé par les faux-semblants et la recherche du plaisir. Dès sa première entrée en scène, Mundo est facilement identifiable comme un Inca car il porte le champi et la mascapaycha (didascalie v. 204). Là encore, le personnage de l'Inca est caractérisé de façon négative, Mundo et la coya Aycha ayant décidé d'abuser le jeune et naïf Cristiano pour l'éloigner de Dieu et le spolier de toutes ses richesses.

Assurément, en connotant négativement la figure de l'Inca, l'auteur de ces autos sacramentales — pièces qui, rappelons-le, étaient à visée didactique — ne faisait que traiter le couple thématique baroque du vanitas vanitatum et du memento mori. Dans ce monde, où tout n'est qu'illusion et apparence et où personne ne peut échapper à la mort, Dieu représente le seul espoir vers lequel se tourner. $C^{\prime}$ est donc en rejetant le passé païen et ses faux semblants, incarnés par la figure de I'Inca et de ses courtisans, et en embrassant résolument les valeurs spirituelles du Christianisme que les Indiens peuvent, à l'instar de Cristiano, trouver leur salut.

La situation change complètement dans les quatre comedias composées au XVIII siècle, c'est-à-dire en pleine Renaissance inca. Les deux Yauri Tito et Usca Paucar revendiquent clairement leur identité de princes incas déchus à la suite de la Conquête ; toutefois, à la différence de Plutón et de Mundo, leur caractérisation

${ }^{12}$ Comme, par exemple, le champi et la maqana (têtes d'armes) affichés par Plutón ou le fait que le roi des Enfers soit accompagné par quatre Manes, nombre qui rappelle celui des gouverneurs des quatre provinces dans lesquelles se divisait le Tahuantinsuyo (Tawantinsuyu litt. « les quatre parties ensemble »). 
est suffisamment positive pour que le public puisse s'identifier à eux. Désespérés par la perte de leurs biens et de leur statut social, ils deviennent une proie facile de Satan qui leur offre amour et richesses en échange de leur âme. Par défaut de connaissances en matière de religion (Yauri Tito dans El pobre más rico) ou, au contraire, en sachant pertinemment qu'ils s'apprêtent à commettre un péché (Usca Paucar dans la pièce homonyme et Yauri Tito dans El milagro del Rosario), ils acceptent de signer un pacte de sang avec le Diable. Mais les plaisirs sensibles ne parviennent pas à leur procurer la satisfaction espérée et, ayant réalisé leur erreur, ils sont sauvés in extremis par l'intervention de la Vierge, qui les arrache des mains de Satan venu recouvrer sa créance.

Certes, la morale de I'histoire n'est pas différente de celle des autos d'Espinosa : les biens matériels sont éphémères, seulement les valeurs spirituelles peuvent apporter le bonheur. Ce qui change, en revanche, c'est que Yauri Tito et Usca Paucar donnent une image très valorisante de I'Inca. Après une période d'égarement - que I'on peut par ailleurs attribuer au grand bouleversement provoqué par la Conquête - , nos protagonistes finissent en effet par se racheter et s'intégrer à la société chrétienne. Comme le fait remarquer Itier, il est significatif que l'action de ces pièces se situe à une époque charnière, entre la Conquête et l'établissement du nouvel ordre colonial. Car il aurait été impensable qu'au XVIII siècle, des représentants de l'élite indigène ignorent les dogmes de la religion catholique, comme c'est le cas pour le protagoniste de El pobre más rico (Itier, 1995 : 95).

Une étape ultérieure est franchie dans Ollantay, dont les protagonistes sont des anciens Incas qui, tout en étant païens, possèdent de hautes valeurs morales. En particulier, le personnage de I'Inca Tupac Yupanqui présente les traits du «Père miséricordieux », qui pardonne à la brébis égarée Ollantay et lui permet de réintégrer une société inca idéalisée, plus imaginaire et mythique que véritablement historique (Martin, 2011a : 123-146). La figure de I'Inca, qui avait déjà été traitée de façon ambiguë par Espinosa13, Centeno et les auteurs des anonymes Usca Paucar et El milagro del Rosario, s'affirme plus que jamais dans Ollantay comme le symbole de la remise en question identitaire réalisée au XVIII siècle par l'élite indigène.

\section{2. Évolution du personnage du gracioso}

À l'instar du noble inca, le gracioso acquiert lui aussi une caractérisation plus positive et un statut plus important dans le passage des autos sacramentales du XVII ${ }^{\mathrm{e}}$ siècle aux comedias du XVIII .

Considérons d'abord l'étendue de parole et la présence en scène du personnage. Dans El robo de Proserpina, Taparaco fait sa première apparition seulement au

\footnotetext{
13 S'il est vrai en effet que, dans El robo de Proserpina, la figure de I'Inca est incarnée par PlutónLucifer, il est vrai également que ce dernier est sans aucun doute le personnage le plus complexe et le plus intéressant de cet auto sacramental. C'est notamment à lui que l'auteur confie le long monologue initial de 382 vers.
} 
vers 536, après que tous les personnages principaux sont déjà entrés en scène. Des 1693 vers dont est constituée la pièce14, lui en sont impartis 264, quantité qui reste significative surtout si I'on considère que El robo de Proserpina n'est pas une comedia mais un auto sacramental. Assurément, Espinosa ne pouvait pas se passer de cet agent du comique qui est, par convention, le seul des personnages à avoir le droit de s'adresser directement au public, quitte à rompre provisoirement l'illusion théâtrale. Son étendue de parole reste cependant faible si nous la comparons à celle de son maître, Plutón, à qui sont attribués 602 vers. Le rôle du gracioso se développe par rapport à celui du protagoniste dans El hijo pródigo, où les deux personnages partagent presque le même nombre des répliques : 282 vers pour Cristiano contre 242 pour Ukhu, sur 1455 qui en compte la pièce15.

Si nous prenons maintenant les trois comedias marianas, nous constatons que la présence en scène du gracioso gagne davantage en ampleur16, au point que c'est à Quespillo que l'auteur de El pobre más rico confie la tâche de prononcer les derniers mots du drame : Kaypitaqmi p'uchukakun / kay inkap kawsayñin. / Wakchamanta qhapaqyaspa / supaypa runanmi karqan. / Kunanri wakcha kaspa / aswan qhapaqmi kapunqa. / Virgen María sirwikuyllam / mana pantay kay pachapi. " C'est ici que se termine / (I'histoire de) la vie de cet Inca / qui, étant devenu riche de pauvre (qu'il était), / fut l'esclave du démon. / Et maintenant qu'il est pauvre, / il sera beaucoup plus riche. / C'est seulement en servant la Vierge Marie / qu'on ne se trompe pas dans ce monde » (El pobre más rico, vv. 3272-3279).

Que la morale de l'histoire soit donnée par Quespillo ne doit pas nous surprendre, car c'est justement du point de vue moral que le gracioso subit la transformation la plus spectaculaire dans le passage des autos sacramentales du XVII siècle aux comedias du XVIII .

Taparaco, notre premier gracioso, est un personnage complètement négatif, sur lequel pèsent de lourds forfaits : c'est notamment lui qui persuade Proserpina à manger les sept graines de goyave qui symbolisent les sept péchés capitaux, en la condamnant ainsi à prolonger son séjour aux Enfers (El robo de Proserpina, vv. 1293-1304). Opportuniste, lâche et fanfaron, il reçoit le châtiment qu'il mérite lorsque Ceres (allégorie de l'Église) le transforme en hibou (vv. 1609-1632).

14 Selon le texte reconstitué par César Itier à partir des manuscrits Cárdenas, Meneses et Navarro (Itier, 2010 : 27-47; 158-217).

15 L'estimation que je donne du nombre de vers impartis au gracioso et du nombre de vers dont sont constitués El hijo pródigo, les trois comedias marianas et Ollantay, est approximative. II existe en effet pour chaque pièce plusieurs manuscrits, le nombre des vers variant de l'un à l'autre. Aucune édition critique, basée sur la reconstitution du texte à partir de tous les manuscrits existants, n'a pour le moment été réalisée.

16780 vers sur les 3279 de El pobre más rico ; 461 vers sur les 1785 de I'Usca Paucar ; 516 vers sur les 2040 de El milagro del Rosario ; 135 vers sur les 1978 d'Ollantay. Piqui Chaqui, pourtant bien dessiné dans le premier acte, est abandonné en cours de route par l'auteur d'Ollantay, drame qui ressent l'influence de nouveaux genres littéraires comme le mélodramme, où il n'y a plus d'espace pour le personnage du gracioso (voir Martin, 2011b : 777-790). 
Plus sympathique, mais tout de même connoté négativement, est Ukhu dans El hijo pródigo. Indissolublement lié à son maître, le Corps est l'illustration par antonomase de la sujétion aux appétits sensuels qui caractérise le personnage du gracioso. Comme nous l'avons vu, Ukhu est surtout un « gros ventre », la gourmandise primant sur ses autres vices. Mais la gourmandise est considérée par l'Église comme l'un des sept péchés capitaux, car elle peut conduire à commettre d'autres péchés. C'est la raison pour laquelle lui revient entièrement la responsabilité d'avoir entraîné son maître sur le mauvais chemin, comme le rappelle le précepteur de Cristiano, Diospa Simin (la « Parole de Dieu ») : Ama Cristiano uyariychu / ukhuykip simi qusqanta. / Awaqaykim pay. Payraykutaqmi / kay ch'ikimanpas chayanki. «N'écoute pas Cristiano / ce que dit ton corps. / II est ton ennemi. / C'est à cause de lui / que tu te trouves dans cette situation » (El hijo pródigo, vv. 1263-1266). C'est également la raison pour laquelle à la fin de la pièce, alors que Cristiano a réintégré la maison de son père et profite des réjouissances organisées en son honneur, Ukhu reçoit une punition exemplaire : écarté de la fête, il est attaché à un poteau avec une tablette de pichqa et une dent suspendues au cou en souvenir du temps passé à la cour de Mundo et au service de Nina Kiru ( «la Dent de Feu ») 17 .

Alors que Taparaco est un opportuniste, lâche et fanfaron et que Ukhu joue le rôle de mauvais conseiller auprès de Cristiano, les graciosos des trois comedias marianas et d'Ollantay sont des confidents avisés, capables d'une fidélité à toute épreuve envers leurs maîtres. Dans le premier acte de El pobre más rico, lorsque Yauri Tito invite Quespillo à partir de son côté car il n'a plus rien à lui offrir à l'exception de son coeur en gage d'amitié, le gracioso refuse catégoriquement de I'abandonner :

\section{Qispillu}

Chayqa chayta ñispalla

sunquyta wiksaykama q'iwirquchin

Manañam ripuymanchu

allquhina waqtasqapas.

\section{Quespillo}

Avec ces mots

tu m'as remué le coeur et les entrailles.

Je ne vais pas m'en aller, même si tu me bat comme un chien!

El pobre más rico, vv. 353-356.

Même dans les adversités et au risque de leur propre vie, nos quatre graciosos restent fidèles à leurs maîtres et affrontent à leur côté la pauvreté, l'exil et toute sorte de dangers. Face au désespoir dans lequel les héros se laissent sombrer, ils réagissent avec détermination. Lorsque Yauri Tito, dans la scène d'exposition de El pobre más rico, se plaint de sa misère et invoque la mort, Quespillo tourne ses propos en dérision et lui impartit une leçon de bon sens :

17 « Ukhu amarrado, con un quiro, y una piscca colgada al cuello : muy triste» (El hijo pródigo, v. 1387). La pichqa est un jeu qui remonte à l'époque précolombienne, que l'on faisait avec une tablette en bois avec des trous et un dé en forme de pyramide tronquée. C'est en jouant au jeu de la pichqa avec Mundo et ses courtisans que Cristiano avait été dépouillé de toutes ses richesses. 


\section{Qispillu}

Rimasqaykikta uyarispa

« astaqa llakisaq » ñinitaq,

hukmantari asikunitaq.

Tukuy hinantin runakunam

wañurikun kawsaymanta.

Qamllapim rikuni kunan

wañunanrayku kawsaqta.

\section{Quespillo}

Quand je t'entends parler, j'ai envie de m'attrister mais en même temps je me ris. Tous les hommes, sans exception, meurent pour vivre.

En toi, pour la première fois, je vois

quelqu'un qui vit pour

mourir.

El pobre más rico, vv. 266-272.

Des mots semblables sont prononcés par les autres deux Quespillo qui, à l'instar de leur modèle littéraire, s'insurgent contre le désir de mort du galán. Car nos graciosos aiment la vie, au point que même leur couardise peut se justifier au nom de cet amour. Et si la vision pragmatique qu'ils ont de l'existence leur vient de la sagesse populaire, le respect et la défense de la vie sont l'un des premiers préceptes de la religion chrétienne. En fustigeant les idées suicidaires de leurs maîtres, nos trois Quespillo se font ainsi les porte-parole des valeurs morales des auteurs. On constate dès lors que les polarités se sont inverties dans le passage des autos sacramentales du XVIIe siècle aux comedias du XVIII e. Ce n'est plus le gracioso qui entraîne le galán sur la mauvaise voie mais c'est désormais le galán, tellement préoccupé à se vautrer dans le désespoir qu'il perd sa foi en Dieu, qui oblige le gracioso à le suivre sur un chemin périlleux. Vox populi et vox Dei, le personnage du serviteur comique devient la conscience du galán.

Bien que dans le premier acte de El pobre más rico ni Yauri Tito ni Quespillo ne connaissent encore bien la religion chrétienne ${ }^{18}$, dès sa première rencontre avec Nina Kiru, le gracioso se méfie de lui et cherche à mettre en garde son maître :

\section{Qispillu}

Yawrit'itu, awkilláy,

haku aykipullasun kaymanta.

Ima runapunich kayqa?

Yaqullan ancha kuyuptinmi

sikinñiqta qhawarini.

Ima manchaytam rikuni :

k'usilluhina chupasapam kasqa,

imamanpas raykukullachwantaq.

El pobre más rico, vv. 722-729

\section{Quespillo}

Yauri Tito, mon cher prince, fuyons vite d'ici !

Quel genre d'homme est-il ?

Quand sa veste s'ouvre,

j'entrevois ses fesses !

Quel spectacle épouvantable :

il a une longue queue comme les singes!

Qu'il ne nous arrive pas quelque malheur!

${ }^{18}$ Comme le témoignent, par exemple, le fait que le gracioso s'enfuit en entendant le chant liturgique du Kyrie Eleison (" Seigneur, aie pitié ») parce qu'il pense que la foule crie auprès de lui k'iriy k'iriy « blesse blesse » (vv. 396-425) ou le fait qu'en voyant un ange, il se demande s'il s'agit d'un gros poulet avec des ailes (vv. 1038-1044). 
Tout ignorant qu'il est de la religion chrétienne, Quespillo ne se trompe pas sur la véritable nature de l'étranger, qu'il compare très judicieusement à un singe car le Diable est «le Singe de Dieu» (Estenssoro, 2001). À la différence de son maître, le gracioso ne se laisse pas séduire par les promesses de Nina Kiru et refuse catégoriquement de signer un pacte avec lui. En essayant d'ouvrir les yeux de Yauri Tito pour qu'il en fasse autant, il renverse totalement les rôles et, en dépit de son traditionnel matérialisme, exhorte son maître à ne pas céder à l'appât de l'argent (El pobre más rico, vv. 783-790).

Que le gracioso agisse comme s'il était la conscience du galán, le témoigne une scène de I'Usca Paucar dans laquelle Quespillo hésite à s'endormir en craignant de laisser son maître livré à lui-même : Ñuqapas puñurqukusaqtaq. / Pim qampaq chupipusunki ? / Picha qampaq ch'arki saqtaq ? / Picha qamta lulusunki ? "Moi aussi je vais dormir. / Mais qui te préparera la soupe ? / Qui cuisinera pour toi de la viande séchée ? / Et qui veillera sur toi ?» (Usca Paucar, vv. 543-546). C'est en effet au moment où Quespillo est endormi que les diables arrivent pour induire en tentation Usca Paucar. II n'y aurait d'ailleurs aucun salut possible pour lui, si Quespillo n'avait pas ramassé et gardé précieusement le rosaire que son maître avait jeté au moment de signer le pacte avec Satan. Ce rosaire deviendra pour Usca Paucar l'instrument de son salut, au moment où les diables et la Vierge se disputeront son âme (Usca Paucar, vv. 1504-1512).

Nous voici en présence d'un personnage de gracioso dont l'intégrité morale est à toute épreuve et dont la foi en Dieu reste intacte en dépit de toutes les adversités, alors que celle du galán vacille au moment où il en aurait le plus besoin. La question qu'on pourrait se poser est de savoir pourquoi les auteurs des comedias marianas ont dévolu la fonction de transmettre le message édifiant de leurs pièces non pas au personnage du noble inca mais au gracioso, qui dans le théâtre quechua colonial incarne l'Indien du commun.

À cette question, nous allons maintenant essayer d'apporter des réponses, en tirant les conclusions des observations réalisées au cours de notre enquête.

Il convient tout d'abord de rappeler que nos investigations n'ont porté que sur un nombre très restreint d'œuvres, les six pièces dont nous disposons ne pouvant pas fournir un cadre exhaustif du théâtre quechua colonial. Pour révélatrices qu'elles soient à nos yeux d'une certaine orientation dans le traitement diachronique du gracioso, il n'en reste pas moins qu'il faut considérer les résultats auquels nous sommes parvenus comme un bilan provisoire, auquel la découverte de nouveaux textes pourrait apporter des éclairages différents. Ajoutons que l'ennoblissement de la figure du gracioso n'est pas un phénomène spécifiquement andin. Les comedias du Siècle d'Or espagnol en présentent de nombreux exemples, comme le témoignent les drames de Juan Ruiz de Alarcón (1581-1639) et, en particulier, la Verdad sospechosa (1618-1621), où le gracioso Tristán constitue un exemple parmi les plus aboutis de caractérisation positive du personnage. D'autre part, enquêter sur les emprunts possibles de nos graciosos andins à tel ou tel homologue espagnol, reviendrait à s'engager dans un long travail comparatif qui n'aurait pas pu se réaliser dans l'espace limité de cet article. De cette revue trop rapide d'un 
corpus sans doute incomplet, il est tout de même possible de dégager un certain nombre de conclusions.

Exemple de transfert culturel réussi, le personnage du gracioso doit sa fortune au sein du théâtre quechua colonial à sa capacité à adapter son riche héritage littéraire aux goûts et aux attentes du public andin. Pour séduire et fidéliser ce public, tout en conservant ses fonctions et ses attributs constitutifs - et en premier lieu sa verve comique - le gracioso n'hésite pas à endosser les traits de l'Indien du commun. II apprend à s'exprimer en quechua à travers un vocabulaire populaire, riche en expressions métaphoriques empruntées à la tradition orale. Il est licite de supposer que le contraste avec le personnage du noble inca, au langage plus recherché et artificiel, devait favoriser sinon l'identification, du moins l'empathie des spectateurs avec cette figure de serviteur aux multiples facettes. L'étendue de parole qui lui est accordée dans les comedias du XVIII ${ }^{\mathrm{e}}$ siècle ainsi que la richesse et l'ambiguïté de ce représentant de la classe populaire, me semblent conforter l'hypothèse que le théâtre quechua colonial s'était popularisé au fil du temps, finissant par gagner toutes les couches de la société cuzquénienne. S'il est donc probable que les autos sacramentales d'Espinosa étaient originairement destinés à être représentés dans le séminaire de San Antonio Abad ou dans le collège pour fils de caciques San Borja de Cuzco devant un public élitaire relativement réduit, il est d'autant plus probable que les drames quechuas du XVIII siècle faisaient l'objet de mises en scène ouvertes à un public populaire. Ce même public auquel Quespillo s'adresse, en le détaillant, dans l'extrait cité en introduction.

Quant au fait que les auteurs des comedias marianas confient au gracioso la tâche de véhiculer les valeurs morales de leurs pièces, une autre explication pourrait être évoquée. II ne fait nul doute que, dans le discours de l'Église, l'homme pauvre est celui qui a la préférence de Dieu, les riches et les puissants étant considérés comme des orgueilleux qui peuvent difficilement prétendre au royaume des cieux. Nos Quespillo auraient ainsi été façonnés à l'image du " pauvre en esprit » des Saintes Écritures, I'homme humble aux mains ouvertes à I'action de Dieu et dont la conduite doit servir de modèle aux riches et puissants au regard altier. Suivant cette clé de lecture, I'histoire de Yauri Tito et d'Usca Paucar est donc à interpréter comme un avertissement donné à l'élite indigène pour qu'elle fasse preuve d'humilité, renonce aux faux semblants et se tourne vers les vraies valeurs. Il s'agit d'un thème récurrent de la littérature religieuse, qui apparaît souvent dans les sermons et dont la présence ne doit pas surprendre dans des pièces de théâtre didactique.

Et I'on pourrait dès lors avancer que, en ennoblissant la figure du gracioso, les auteurs des drames quechuas coloniaux se proposaient de montrer que même les Indiens du commun, en dépit de leur simplicité — ou plutôt en vertu de celleci —, étaient dotés d'une spiritualité sincère et devaient donc être considérés comme des membres à part entière de la communauté des croyants. II ne faut pas oublier, à ce propos, que l'accès aux ordres religieux fut longtemps refusé aux Indiens sous prétexte qu'ils étaient des néophytes encore chancelants qu'il fallait soutenir et guider. L'idéologie coloniale ne pouvait pas les considérer comme entièrement convertis, du moment que la Conquête et I'occupation espagnole 
trouvaient leur justification dans la nécessité d'évangéliser des peuples idolâtres. Permettre aux Indiens l'accès au sacerdoce aurait signifié leur donner une pleine autonomie religieuse et donc admettre que la présence espagnole en Amérique n'était plus nécessaire (Estenssoro, $2003: 142-143$ ).

En tant que porte-parole des revendications sociales et politiques de l'élite indigène, les auteurs des comedias du XVIII e siècle prirent parti pour leurs causes, dont celle de l'accès aux ordres religieux, qui est peut-être inscrite en filigrane dans les comedias marianas. Conscients de l'efficacité dramatique et de la popularité du gracioso, ils firent de lui un personnage-clé, à qui était dévolue la tâche de légitimer par son exemple les revendications du groupe social à qui étaient destinés les drames du théâtre quechua colonial et de satisfaire, en même temps, les attentes du public populaire dont il était le digne représentant.

\section{Références citées}

ALAPERRINE-BOUYER, M., 2007 - La educación de la elites indígenas en el Perú colonial, 345 p. ; Lima : Institut Français d'Études Andines (IFEA), Instituto Riva-Agüero.

ANÓNIMO, 1554 - La vida de Lazarillo de Tormes y de sus fortuna y adversidades; Medina del Campo: Mateo y Francisco del Campo.

BURGA DÍAZ, M., 1988 - Nacimiento de una utopía: muerte y resurrección de los Incas, 428 p. ; Lima : Instituto de Apoyo Agrario.

CHANG-RODRÍGUEZ, R., 1991 - Salvación y sumisión en el Usca Paucar. In : El discurso disidente. Ensayos de literatura colonial peruana : 208-245 ; Lima : Pontificia Universidad Católica del Perú (PUCP).

CISNEROS, L. J. \& GUIBOVICH PÉREZ, P., 1988 - Juan de Espinosa Medrano, un intelectual cuzqueño del seiscientos : nuevos datos biográficos. Revista de Indias, 48 (182183) : 327-347.

DUMAS, C., 2004 - Du gracioso au valet comique. Contribution à la comparaison de deux dramaturgies (1610-1660), 437 p. ; Paris : Honoré Champion Éditeur.

ESTENSSORO FUCHS, J. C., 2001 - El simio de Dios. Los indígenas y la Iglesia frente a la evangelisación del Perú, siglos XVI-XVII. Bulletin de I'Institut Français d'Études Andines, 30 (3) : 455-474.

ESTENSSORO FUCHS, J. C., 2003 - Del paganismo a la santidad. La incorporación de los indios del Perú al catolicismo 1532-1750, 586 p. ; Lima : Institut Français d'Études Andines (IFEA), Instituto Riva-Agüero-Pontificia Universidad Católica del Perú (PUCP).

FLORES ESPINOZA, J., 2001 - La añoranza del pasado. Justo Sahuaraura inca y sus «Recuerdos de la monarquía peruana». Estudio introductivo a Don Justo Apu Sahuaraura Inca, Recuerdos de la monarquía peruana o Bosquejo de la historia de los Incas [1838] : 13-46 ; Lima : Fundación Telefónica del Perú. 
GARCÍA-BEDOYA, C., 1998 - Teatro quechua colonial, barroco andino y renacimiento inca. In : Actas del I Encuentro Internacional de Peruanistas: 325-338 ; Lima : Universidad de Lima, 3-6 de septiembre 1996.

GARCÍA-BEDOYA, C., 2000 - La literatura peruana en el periodo de estabilización colonial (1580-1780), 300 p ; Lima : Universidad Nacional Mayor de San Marcos (UNMSM).

GOMEZ, J., 2006 - La figura del donaire o el gracioso en las comedias de Lope de Vega, 141 p. ; Sevilla : Ediciones Alfar.

ITIER, C., 1995 - Quechua y cultura en el Cuzco del siglo XVIII. De la 'lengua general' al 'idioma del imperio de los Incas'. In : Del siglo de oro al siglo de las luces. Lenguaje y sociedad en los Andes del siglo XVIII : 89-111; Cuzco : Centro de Estudios Regionales Andinos 'Bartolomé de Las Casas'.

ITIER, C., 2006 - Ollantay, Antonio Valdez y la rebelión de Thupa Amaru. Historica, 30 (1) : 65-97.

ITIER, C., 2010 - El robo de Proserpina y sueño de Endimión. Auto sacramental en quechua, 217 p. ; Lima : Institut Français d'Études Andines (IFEA), Pontificia Universidad Católica del Perú (PUCP).

LIENHARD, M., 1992 - La voz y su huella. Escritura y conflicto étnico-cultural en América Latina 1492-1988 (3a ed. rev. y aum.), 305 p. ; Lima : Horizonte.

MANNHEIM, B., 1990 - La cronología relativa de la lengua y literatura quechua cusqueña. Revista andina, 8 (1) : 139-184.

MARTIN, R., 2007 - Ollantay. Trajectoire d'un mythe littéraire andin (XVIII - XXI e siècles). Thèse de doctorat soutenue à I'Institut national de Langues et Civilisations orientales (INALCO).

MARTIN, R., 2011a - Tupac Yupanqui ou le modèle du prince parfait. Étude de l'autre protagoniste d'Ollantay. Bulletin de l'Institut Français d'Études Andines, 40 (1) : 123 146.

MARTIN, R., 2011b - La représentation de l'ancien Tawantinsuyu dans une utopie andine du XVIII siècle. In : Quaderni di Thule. Rivista italiana di studi americanistici. Atti del XXXII Convegno Internazionale di Americanistica (Perugia, 4-10 maggio 2010) : 777-790 ; Perugia, Centro Studi Americanistici « Circolo Amerindiano » Onlus, X.

MENESES, T. L., 1977 - Datación y paternidad del drama "Apu Ollantay". Revista de Arte, Ciencias y Humanidades, 17 : 49-82 ; Lima : Universidad Nacional Mayor de San Marcos (UNMSM) Fondo Editorial.

MENESES, T. L., 1983 - Teatro quechua colonial. Antología, 593 p. ; Lima : Ediciones Edubanco.

PORRAS BARRENECHEA, R., 1954a - El padre Valdez, autor del Ollantay. El Comercio, Lima, 18-11-1954. Réédité in Indagaciones peruanas. El legado quechua (Raúl Porras Barrenechea, ed.) : 390-393 ; Lima : Instituto Raúl Porras BarrenecheaUniversidad Nacional Mayor de San Marcos (UNMSM) Fondo Editorial.

PORRAS BARRENECHEA, R., 1954b - La paternidad definitiva de Ollantay. El Comercio, Lima, 19-11-1954. Réédité in Indagaciones peruanas. El legado quechua (Raúl Porras Barrenechea, ed.) : 393-396 ; Lima : Instituto Raúl Porras BarrenecheaUniversidad Nacional Mayor de San Marcos (UNMSM) Fondo Editorial.

PORRAS BARRENECHEA, R., 1955 - El Ollantay y Antonio Valdez. El Comercio, Lima, 17-11-1955. Réédité in Indagaciones peruanas. El legado quechua (Raúl Porras Barrenechea, ed.) : 396-399 ; Lima : Instituto Raúl Porras Barrenechea-Universidad Nacional Mayor de San Marcos (UNMSM) Fondo Editorial. 
La figure du gracioso dans le théâtre quechua colonial

RODRÍGUEZ GARRIDO, J. A., 1994 - Retórica y tomismo en Espinosa Medrano. Cuadernos de investigación, 3 : 32.

ROWE, J. H., 1954 - El movimiento nacional inca del siglo XVIII. Revista Universitaria, Cuzco, XLIII (107) : 17-47.

VALDEZ, A., 1938 - Ollantay. Ad fidem Codicis Pastor-Justinianiensis. Archivii Nationalis Limensis recensuit. Latine vertit. Integra Codicis Sahuaraurensis lectione. Analysi morphologica grammatica indicibus dissertationibus scholiis auxit Prof. Dr. Hippolytus Galante : 13-44 (fac-similé du manuscrit Justiniani), 73-149 (transcription et traduction latine de l'acte I) ; Lima : Publicaciones del Instituto Superior de lingüística y filología de la Universidad Mayor de San Marcos - Monumenta Linguae Incaicae.

VITULLI, J. M., 2011 - Amar su propia muerte, 185 p. ; Madrid : Iberoamericana - Vervuert y Consejo Superior de Investigaciones Científicas. 